NASA/TM-2008-215312

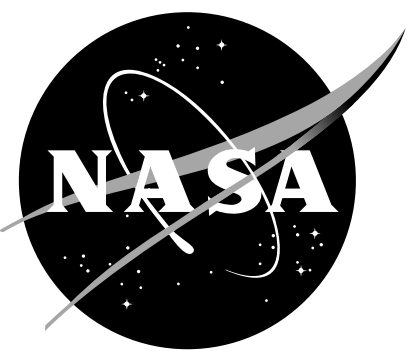

\title{
Allowable Trajectory Variations for Space Shuttle Orbiter Entry-Aeroheating CFD
}

William A. Wood and Stephen J. Alter

Langley Research Center, Hampton, Virginia 
Since its founding, NASA has been dedicated to the advancement of aeronautics and space science. The NASA Scientific and Technical Information (STI) Program Office plays a key part in helping NASA maintain this important role.

The NASA STI Program Office is operated by Langley Research Center, the lead center for NASA's scientific and technical information. The NASA STI Program Office provides access to the NASA STI Database, the largest collection of aeronautical and space science STI in the world. The Program Office is also NASA's institutional mechanism for disseminating the results of its research and development activities. These results are published by NASA in the NASA STI Report Series, which includes the following report types:

- TECHNICAL PUBLICATION. Reports of completed research or a major significant phase of research that present the results of NASA programs and include extensive data or theoretical analysis. Includes compilations of significant scientific and technical data and information deemed to be of continuing reference value. NASA counterpart of peer-reviewed formal professional papers, but having less stringent limitations on manuscript length and extent of graphic presentations.

- TECHNICAL MEMORANDUM. Scientific and technical findings that are preliminary or of specialized interest, e.g., quick release reports, working papers, and bibliographies that contain minimal annotation. Does not contain extensive analysis.

- CONTRACTOR REPORT. Scientific and technical findings by NASA-sponsored contractors and grantees.
- CONFERENCE PUBLICATION. Collected papers from scientific and technical conferences, symposia, seminars, or other meetings sponsored or co-sponsored by NASA.

- SPECIAL PUBLICATION. Scientific, technical, or historical information from NASA programs, projects, and missions, often concerned with subjects having substantial public interest.

- TECHNICAL TRANSLATION. Englishlanguage translations of foreign scientific and technical material pertinent to NASA's mission.

Specialized services that complement the STI Program Office's diverse offerings include creating custom thesauri, building customized databases, organizing and publishing research results ... even providing videos.

For more information about the NASA STI Program Office, see the following:

- Access the NASA STI Program Home Page at http://www.sti.nasa.gov

- E-mail your question via the Internet to help@sti.nasa.gov

- Fax your question to the NASA STI Help Desk at (301) 621-0134

- Phone the NASA STI Help Desk at (301) 621-0390

- Write to: NASA STI Help Desk NASA Center for AeroSpace Information 7115 Standard Drive Hanover, MD 21076-1320 
NASA/TM-2008-215312

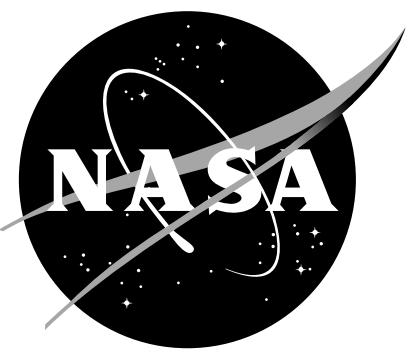

\section{Allowable Trajectory Variations for Space Shuttle Orbiter Entry-Aeroheating CFD}

William A. Wood and Stephen J. Alter

Langley Research Center, Hampton, Virginia 
The use of trademarks or names of manufacturers in this report is for accurate reporting and does not constitute an offical endorsement, either expressed or implied, of such products or manufacturers by the National Aeronautics and Space Administration.

Available from:

NASA Center for AeroSpace Information (CASI)

7115 Standard Drive

Hanover, MD 21076-1320

(301) 621-0390

National Technical Information Service (NTIS) 5285 Port Royal Road

Springfield, VA 22161-2171

(703) 605-6000 


\begin{abstract}
Reynolds-number criteria are developed for acceptable variations in Space Shuttle Orbiter entry trajectories for use in computational aeroheating analyses. The criteria determine if an existing computational fluid dynamics solution for a particular trajectory can be extrapolated to a different trajectory. The criteria development begins by estimating uncertainties for seventeen types of computational aeroheating data, such as boundary layer thickness, at exact trajectory conditions. For each type of datum, the allowable uncertainty contribution due to trajectory variation is set to be half of the value of the estimated exact-trajectory uncertainty. Then, for the twelve highest-priority datum types, Reynolds-number relations between trajectory variation and output uncertainty are determined. From these relations the criteria are established for the maximum allowable trajectory variations. The most restrictive criterion allows a $25 \%$ variation in Reynolds number at constant Mach number between trajectories.
\end{abstract}

\title{
1 Nomenclature
}

The subject matter in the present report considers relative changes in fluid dynamic properties. Therefore dimensional units for the symbols are not pertinent to the results.

\section{Symbols}

$\begin{array}{ll}C_{P} & \text { Pressure coefficient } \\ D & \text { Cavity depth } \\ H & \text { Total enthalpy } \\ M & \text { Mach number } \\ P & \text { Pressure } \\ q & \text { Heat transfer rate } \\ R e & \text { Reynolds number } \\ T & \text { Temperature } \\ V & \text { Velocity magnitude } \\ \delta & \text { Boundary layer thickness } \\ \delta^{*} & \text { Displacement thickness } \\ \mu & \text { Viscosity } \\ \rho & \text { Density } \\ \theta & \text { Momentum thickness }\end{array}$

\section{Subscripts}

e $\quad$ Boundary layer edge 


$\begin{array}{ll}k & \text { Protuberance height } \\ w & \text { Wall } \\ \infty & \text { Free stream }\end{array}$

\section{Operators}

$\Delta \quad$ Difference: $\Delta x=x_{2}-x_{1}$

\section{Acronyms}

BF Bump factor

BLT Boundary layer transition

CFD Computational fluid dynamics

DPLR Data-parallel line relaxation CFD code

LAURA Langley aerothermodynamic upwind relaxation algorithm CFD code OML Outer mold line

RCC Reinforced carbon-carbon

\section{Introduction}

The Space Shuttle Orbiter Entry Aeroheating Subsystem team has pre-computed computational fluid dynamics (CFD) solutions of Orbiter flow fields for nominal entry trajectories [1]. During missions, these solutions may be used to predict aerothermodynamic properties in the course of assessing Orbiter damage and repairs [2]. But, the mission-specific entry trajectory will not match the nominal trajectory, because of differences such as the Orbiter weight or atmospheric conditions. These differences in trajectories can be expressed as differences in Reynolds numbers at given Mach numbers. This report develops Reynolds-number criteria to characterize how close in trajectory space a particular trajectory must be to a nominal trajectory so that the nominal CFD solutions are sufficient for damage assessment purposes. The most restrictive criterion is then used to define the allowable trajectory variations for reuse of the nominal CFD solutions during a mission.

The criteria are developed under several assumptions: a spot-check at one Mach number applies for all Mach numbers (the Mach numbers of most interest are greater than 15); uncertainties are symmetric and can be expressed as \pm quantities; uncertainty trends extrapolate linearly in Reynolds number; and, angle of attack variations are negligible (the angles of attack are within $40 \pm 2^{\circ}$ ).

The development of the criteria begins by identifying seventeen types of aerothermodynamic data, such as surface temperature and boundary layer thickness, that are available in the CFD solution set and are relevant to Orbiter damage or repair assessments. For each datum type, uncertainty estimates or accuracy requirements are obtained for the nominal trajectory cases, at 
approximately 1:20 odds. Then the allowable uncertainty contribution due to trajectory variations is defined to be one-half of the uncertainty estimate from the previous step. The seventeen types of data are prioritized, and, because of resource limitations, criteria are pursued for only the top twelve types. Reynolds number scalings are then developed to relate trajectory variations to variations in the CFD results. Finally, the allowable trajectory Reynolds number variations are determined by inverting the scaling relations.

The Reynolds number criteria are summarized as a tabulation in section 4.14 .

\section{Nominal Orbiter CFD solutions}

Aeroheating CFD solutions of entry flow fields about the Orbiter vehicle ${ }^{1}$ exist for several points along a few nominal trajectories. The CFD codes employed, the solution processes, the verification techniques, the validation data, and the solutions themselves have been accepted for use during Space Shuttle missions by the Orbiter Configuration Control Board [1]. Previous Orbiter solutions of a similar nature and application, using the same CFD codes, have been reported [3] in the context of the Columbia accident investigation.

These solutions typically require about a week each to obtain, although with exclusive access to a supercomputer in a round-the-clock staffing mode solutions have been obtained in a day or two. It is desired to avoid this cost of creating new entry Orbiter solutions; thus the present report's topic of how much these existing solutions can be reused for different trajectories.

\subsection{CFD codes}

Two CFD codes are employed for the present Orbiter entry aeroheating analyses. The use of two codes provides a measure of verification when used redundantly, and pragmatically allows the team members to choose their most proficient analysis tool. The two codes are the Data Parallel Line-Relaxation (DPLR) software [4] and the Langley Aerothermodynamic Upwind Relaxation Algorithm (LAURA) [5,6].

Both DPLR and LAURA are finite-volume upwind-flux schemes for solving the Navier-Stokes $[7,8]$ equations with non-equilibrium chemistry. All the CFD results considered here have laminar boundary layers, a five-species air model, and assume a radiative-equilibrium surface temperature using reaction-cured glass properties.

\footnotetext{
${ }^{1}$ The CFD Orbiter model used by the Entry Aeroheating Subsystem team is truncated in the vicinity of the body-flap hinge line, 1280 inches from the nose.
} 


\begin{tabular}{cl} 
Priority & Parameter \\
\hline 1 & Idealized cavity heating bump factors \\
2 & Smooth OML surface temperatures \\
3 & Smooth OML heating rates \\
4 & Boundary layer thickness \\
5 & $\frac{R e_{\theta}}{M_{e}} \cdot \frac{1}{\delta} \cdot\left(\frac{H_{e}}{H_{w}}\right)^{0.3}-$ BLT correlation parameter \\
6 & $R e_{k}^{0.6}\left(R e_{\theta} \cdot \frac{\mu_{e}}{\mu_{k}}\right)^{0.4}$ - BLT correlation parameter \\
7 & $\frac{R e_{\theta}}{M_{e}} \cdot \frac{1}{\delta} \cdot\left(\frac{T_{e}}{T_{w}}\right)^{0.51}-$ BLT correlation parameter \\
8 & $\frac{R e_{\theta}}{M_{e}} \cdot \frac{1}{\delta} \cdot\left(\frac{T_{e}}{T_{w}}\right)^{0.67}-$ BLT correlation parameter \\
9 & Boundary layer $C_{p}$, shock relations \\
10 & Boundary layer $C_{p}$, stream-tube relations \\
11 & Displacement thickness \\
12 & $\frac{H_{w}}{H_{e}}$ \\
13 & Protruding gap-filler $C_{p}$ \\
14 & RCC plug/NOAX heating bump factors \\
15 & Wing leading-edge heating rates \\
16 & Lee-side heating rates \\
17 & Turbulent heating rates
\end{tabular}

Table 1. CFD aeroheating products, ordered by perceived importance.

\subsection{Solution repository}

There are currently 20 full-vehicle Orbiter solutions, stored in a NASA data repository, covering parts of three nominal trajectories. The solutions span the Mach number range 6-25. The trajectories correspond to STS-107 (Columbia's last flight), STS-115 (a typical entry from the International Space Station), and ISSHVFW (a severe entry from the International Space Station).

\section{Trajectory variation criteria}

Seventeen CFD aeroheating products are considered. The products are listed in table 1 as prioritized by the Entry Aeroheating Subsystem team, based on the perceived importance for mission-support assessments. Except for the "lee-side heating rates," the products are considered by this report only on the wind side of the Orbiter.

For each product, the following sections define the product, explain its application for mission support, and present either an uncertainty estimate or an accuracy requirement for the CFD prediction based upon a nominal trajectory point. Then, the allowable error contribution due to using a nominal-trajectory CFD solution for an off-nominal trajectory point is set to be half of the uncer- 
tainty estimate or accuracy requirement. For example, if drag is a product of interest, and the CFD is estimated to predict drag within $\pm 50 \%$ for a nominal trajectory point, then the trajectory is allowed to be off-nominal up to the point that drag changes by $25 \%$ from the nominal value. For this hypothetical drag example, the root-sum-square total uncertainty would then be capped at $\sqrt{50^{2}+25^{2}}= \pm 56 \%$, assuming a maximal-allowed off-nominal trajectory. In general, this approach caps the the total uncertainty growth by a factor of 0.12 over the nominal-trajectory uncertainty.

For the twelve highest-priority products, the Reynolds number is used to parameterize the degree a trajectory is off-nominal, and criteria are developed that relate trajectory Reynolds number variation to the allowable error in the aeroheating product due to trajectory variation from nominal. The criteria limits are set in the context of approximately 1:20 odds. These criteria are developed subject to several assumptions. Usually, spot-checks are only performed at one Mach number, with the criteria assumed to apply for all Mach numbers; the Mach numbers of most interest are 15-25. Variations are most often only considered in one direction, and the rates of change are assumed to be the same in both the positive and negative sides. The product variations are assumed to extrapolate linearly with Reynolds number. The effects of angle of attack variations are assumed to be negligible; the angles of attack are within $40 \pm 2^{\circ}$.

The trajectory criteria are summarized in table 3 in section 4.14 .

\subsection{Cavity heating bump factors}

The cavity heating bump factors are surface heat transfer rates that have been normalized to a reference nominal heating rate on the smooth OML. The cavities are simplified models of actual damage geometries, such as hexahedrons, and solutions are obtained as local perturbations within the global Orbiter flow field. In the terminology of the Entry Aeroheating Subsystem team, these geometry models are called idealized cavities. Examples of the cavity modeling and analysis process have been published by Pulsonetti and Wood [9]. CFD cavity heating bump factors can be used during missions to assess and supplement the predictions from the Cavity Heating Tool [10], which is the standard cavity heating engineering tool used by the Entry Aeroheating Subsystem team. Of primary interest is the predicted bump factors on the floor of a cavity.

A validation of the CFD cavity heating bump factors [11] has been performed, and the uncertainty estimate for the CFD results is $\pm 50 \%$. The allowable error in bump factors due to trajectory variations is set to be $\pm 25 \%$.

The trajectory criteria for cavity bump factors is developed using the Cav-

ity Heating Tool correlations, which are based upon cavity wind tunnel data. In particular, two of the Cavity Heating Tool correlations show a sensitivity 
to free stream conditions: the fore- $85 \%$ floor of Everhart (short) cavities and the aft-10\% floor of long cavities. See reference [10] for a full description of the Cavity Heating Tool nomenclature.

The Everhart cavity fore- $85 \%$ floor bump factor varies with boundary layer thickness as

$$
B F=\frac{5}{4}-\frac{5}{2} \frac{D}{\delta}, \quad 0.1<\frac{D}{\delta}<0.3
$$

The difference in bump factor for the same cavity at two different boundary layer thicknesses can be expressed in absolute and relative terms as

$$
\begin{gathered}
\triangle B F=B F_{2}-B F_{1} \\
\Delta B F=-\frac{5}{2} \frac{D}{\delta_{1}}\left(\frac{\delta_{1}}{\delta_{2}}-1\right) \\
\frac{\Delta B F}{B F_{1}}=\frac{\frac{\delta_{1}}{\delta_{2}}-1}{1-\frac{1}{2\left(D / \delta_{1}\right)}}
\end{gathered}
$$

Similarly, the long cavity aft-10\% floor bump factor varies with boundary layer thickness as

$$
B F=\frac{14}{3} \frac{D}{\delta}-\frac{3}{5}, \quad 0.3<\frac{D}{\delta}<1.2
$$

The absolute and relative changes in bump factor as functions of boundary layer thickness are

$$
\begin{gathered}
\Delta B F=\frac{14}{3} \frac{D}{\delta_{1}}\left(\frac{\delta_{1}}{\delta_{2}}-1\right) \\
\frac{\Delta B F}{B F_{1}}=\frac{\frac{\delta_{1}}{\delta_{2}}-1}{1-\frac{9}{70\left(D / \delta_{1}\right)}}
\end{gathered}
$$

Variations in boundary layer thickness can be related to variations in Reynolds number, because the boundary layer thickness varies approximately linearly with the reciprocal of the square root of the Reynolds number, as demonstrated in section 4.4. This relationship is expressed as

$$
\begin{gathered}
\delta \propto \frac{1}{\sqrt{R e}} \\
\frac{\delta_{1}}{\delta_{2}}=\sqrt{\frac{R e_{2}}{R e_{1}}}
\end{gathered}
$$

Now, the change in bump factors can be expressed in terms of Reynolds number variations, and so can the inverse relations for Reynolds number 
change be expressed in terms of bump factor variations. For the Everhartcavity fore- $85 \%$ floor bump factor

$$
\begin{gathered}
\Delta B F=-\frac{5}{2} \frac{D}{\delta_{1}}\left(\sqrt{\frac{R e_{2}}{R e_{1}}}-1\right) \\
\frac{R e_{2}}{R e_{1}}=\left(1-\frac{2 \Delta B F}{5\left(D / \delta_{1}\right)}\right)^{2} \\
\frac{\Delta B F}{B F_{1}}=\frac{\sqrt{\frac{R e_{2}}{R e_{1}}}-1}{1-\frac{1}{2\left(D / \delta_{1}\right)}} \\
\frac{R e_{2}}{R e_{1}}=\left[1+\left(1-\frac{1}{2\left(D / \delta_{1}\right)}\right) \frac{\Delta B F}{B F_{1}}\right]^{2}
\end{gathered}
$$

For the long-cavity aft-10\% floor bump factor

$$
\begin{gathered}
\Delta B F=\frac{14}{3} \frac{D}{\delta_{1}}\left(\sqrt{\frac{R e_{2}}{R e_{1}}}-1\right) \\
\frac{R e_{2}}{R e_{1}}=\left(1+\frac{3 \Delta B F}{14\left(D / \delta_{1}\right)}\right)^{2} \\
\frac{\Delta B F}{B F_{1}}=\frac{\sqrt{\frac{R e_{2}}{R e_{1}}}-1}{1-\frac{9}{70\left(D / \delta_{1}\right)}} \\
\frac{R e_{2}}{R e_{1}}=\left[1+\left(1-\frac{9}{70\left(D / \delta_{1}\right)}\right) \frac{\Delta B F}{B F_{1}}\right]^{2}
\end{gathered}
$$

Because equations $11,13,15$, and 17 are monotonic in $\delta_{1}$, a positive-definite quantity, the extreme value theorem states that the maximum variation will occur at an endpoint of the $\delta$ ranges listed in equations 1 and 5 .

By equation $1, B F(D / \delta=0.1)=1$. Using either equation 11 or 13 , it can be determined that a $300 \%$ increase in Reynolds number is needed to reduce the bump factor by $25 \%$ to 0.75 .

By equation 1, $B F(D / \delta=0.3)=0.5$. Using either equation 11 or 13 , it can be determined that a $31 \%$ decrease in Reynolds number is needed to increase the bump factor by $25 \%$ to 0.625 .

By equation $5, B F(D / \delta=0.3)=0.8$. Using either equation 15 or 17 , it can be determined that a $31 \%$ increase in Reynolds number is needed to increase the bump factor by $25 \%$ to 1.0 .

By equation $5, B F(D / \delta=1.2)=5$. Using either equation 15 or 17 , it can be determined that a $40 \%$ decrease in Reynolds number is needed to decrease the bump factor by $25 \%$ to 3.75 . 
Coincidentally, the limiting allowable variation in Reynolds number to produce a $25 \%$ variation in bump factor is the same for both the Everhart and long cavities - $31 \%$. Thus the cavity heating bump factor criteria for allowable trajectory variation is a $\pm 31 \%$ variation in Reynolds number.

\subsection{Surface temperature}

The CFD surface temperatures are radiative equilibrium values assuming reaction-cured glass properties for the entire Orbiter. Of primary interest is the wind-side tile acreage of the vehicle. The CFD surface temperatures may be used during a mission to assess the predicted values from the standard entry aeroheating tools.

A validation of CFD surface temperatures against measured Orbiter flight data reported a two-sigma uncertainty estimate of $\pm 6 \%$ [1]. The allowable change in surface temperature predictions due to trajectory variations is therefore set to $\pm 3 \%$.

The variation in surface temperature with free stream conditions has been approximately scaled by $\left(\rho_{\infty} V_{\infty}^{2}\right)^{1 / 8}$ for an Orbiter boundary layer probe tool [12]. But the present analysis is considering variations in Reynolds number at a fixed Mach number. For the Orbiter entry trajectories, because the temperature does not vary much in the mesosphere, the possible velocity variation at a fixed Mach number is small. Thus, the Reynolds number variations are caused primarily due to density variations at different altitudes, and the surface temperature changes can be related to Reynolds number variations scaled to the $1 / 8$ power.

The Mach-18 STS-115 trajectory point has a 21.1\% lower Reynolds number than the Mach-18 STS-107 trajectory point. Multiplying the Mach-18 STS-107 nominal surface temperatures by the Reynolds number ratio to the $1 / 8$ power, $0.789^{(1 / 8)}=0.971$, produces an estimate of the STS-115 surface temperatures. The error in this estimate relative to the nominal STS-115 solution, for the wind side of the Orbiter, is shown in figure 1 . The errors are within $\pm 2 \%$ over at least $95 \%$ of the surface.

Using a $\pm 2 \%$ bound on the error, the Reynolds number criterion for allowable trajectory variation of surface temperatures is

$$
\left(\frac{3 \% \text { allowable }}{2 \% \text { observed }}\right) 21.1 \%=31.7 \% \simeq \pm 32 \%
$$

\subsection{Smooth OML heating}

The CFD surface heat transfer rates are based upon reaction-cured glass properties for the entire Orbiter. Of primary interest is the wind-side tile acreage of the vehicle. The CFD smooth-OML heating rates may be used during a 


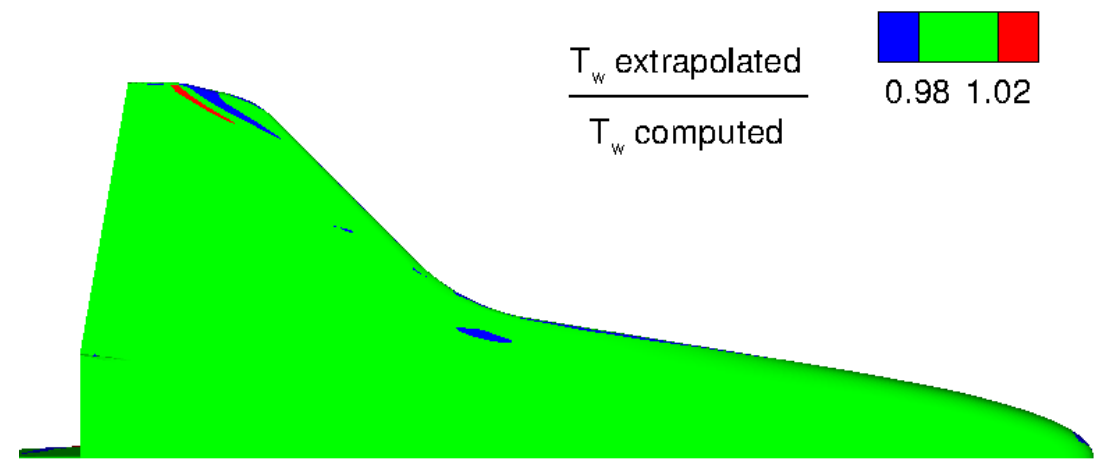

Figure 1. Extrapolation error in surface temperature for Mach-18 check case.

mission to assess the predicted values from the standard entry aeroheating tools.

A validation of CFD surface heating rates against measured Orbiter flight data reported a two-sigma uncertainty estimate of $\pm 20 \%$ [1]. The allowable change in heat transfer rate predictions due to trajectory variations is set to $\pm 10 \%$.

In section 4.2 , the surface temperatures are shown to scale with $R e^{(1 / 8)}$. For radiative equilibrium the relation between surface temperature and heating rates is $q \propto T^{4}$. The assumed variation in heating due to Reynolds number changes is then $q \propto \sqrt{R e}$.

The Mach-18 STS-115 trajectory point has a $21.1 \%$ lower Reynolds number than the Mach-18 STS-107 trajectory point. Multiplying the Mach-18 STS107 nominal surface heating rates by $\sqrt{0.789}=0.888$ produces an estimate of the STS-115 heating rates. The error in this estimate relative to the nominal STS-115 solution, for the wind side of the Orbiter, is shown in figure 2. The errors are within $\pm 7 \%$ over about $95 \%$ of the surface.

Using a $\pm 7 \%$ bound on the error, the Reynolds number criterion for allowable trajectory variation of smooth-OML surface heating rates is

$$
\left(\frac{10}{7}\right) 21.1 \%=30.1 \% \simeq \pm 30 \%
$$

\subsection{Boundary layer thickness}

The CFD boundary layer thickness has been defined by the Entry Aeroheating Subsystem team for the Orbiter flow fields as a variation on the traditional $99.5 \% H / H_{\infty}$ definition. See references $[1,12,13]$ for details of the boundary layer thickness definition. The boundary layer thickness may be used as a 


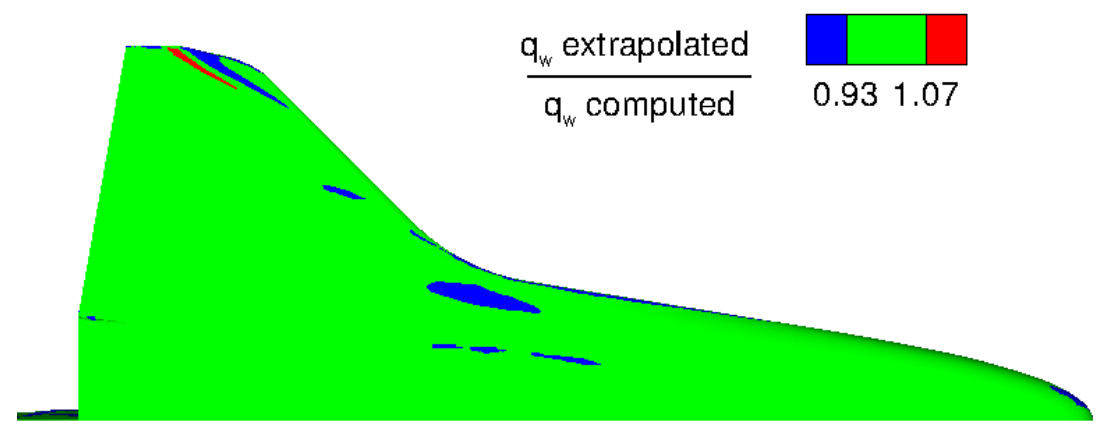

Figure 2. Extrapolation error in heating rates for Mach-18 check case.

\begin{tabular}{ccc}
$M_{\infty}$ & $\frac{R e_{115}-R e_{107}}{R e_{107}}$ & $\sqrt{\frac{R e_{107}}{R e_{115}}}$ \\
\hline 24.9 & $19.6 \%$ & 0.91 \\
24.2 & 6.2 & 0.97 \\
22.9 & -9.4 & 1.05 \\
20.3 & -7.1 & 1.04 \\
19.4 & -16.7 & 1.10 \\
17.9 & -21.1 & 1.13
\end{tabular}

Table 2. Boundary layer thickness scaling factors from STS-107 to STS-115 trajectories.

correlating parameter when creating engineering models of Orbiter damage during a mission.

A validation of CFD boundary layer thicknesses against measured wind tunnel data on an Orbiter model reported a two-sigma uncertainty estimate of $\pm 22 \%$ [1]. The allowable change in boundary layer thickness predictions due to trajectory variations is set to $\pm 11 \%$.

Classic incompressible boundary layer theory [14] scales boundary layer thickness inversely with $\sqrt{R e}$. A $1 / \sqrt{R e}$ scaling was used to extrapolate boundary layer thicknesses at three locations on the Orbiter - at the nose landing gear door, at the external tank door, and at the RCC-9 carrier panelfrom the STS-107 trajectory to the STS-115 trajectory for six trajectory points spanning Mach 17.9-24.9. The scaling factors are listed in table 2.

Figure 3 plots the absolute value of the extrapolation error, relative to CFD solutions for the STS-115 free-stream conditions, for each of the eighteen check points versus the absolute value of the percentage variation in Reynolds number between the STS-107 and STS-115 trajectories. No simple correlation is evident, but the errors are bounded by about $5.5 \%$, with a maximum $21 \%$ 


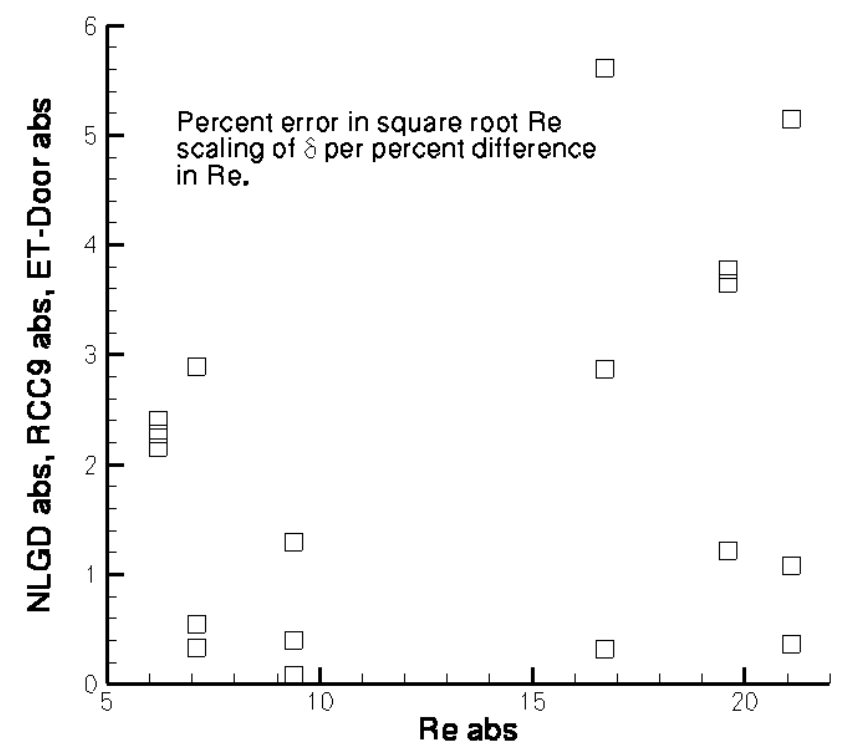

Figure 3. Extrapolation errors for boundary layer thickness test points.

variation in Reynolds number.

The Reynolds number criterion for allowable trajectory variation of boundary layer thicknesses is

$$
\left(\frac{11}{5.5}\right) \times 21.1 \%=42.2 \% \simeq \pm 40 \%
$$

\subsection{Protuberance boundary layer trip parameter}

As part of the primary correlation for predicting boundary layer transition induced by a protuberance, the Entry Aeroheating Subsystem team uses the parameter

$$
\frac{R e_{\theta}}{M_{e}} \cdot \frac{1}{\delta} \cdot\left(\frac{H_{e}}{H_{w}}\right)^{0.3}
$$

All of the variables in parameter (21) are obtained from CFD solutions of the smooth-OML Orbiter. The scaling of this parameter leads to the most restrictive Reynolds number trajectory criterion.

As part of the Boundary Layer Transition Tool (version 2) documentation, a requirement of $11 \%$ accuracy to one standard deviation odds was placed on the CFD data [15]. Extrapolating this accuracy requirement to two-sigma, or approximately $95 \%$, odds yields an accuracy requirement of approximately $20 \%$. The allowable error contribution due to trajectory variations is then set at $10 \%$. 


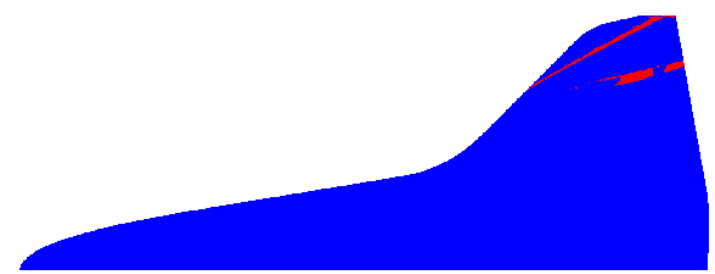

Figure 4. Absolute values of percentage extrapolation errors for protuberance boundary layer transition parameter $(21)$. Red is more than $6.5 \%$. Blue is less than $6.5 \%$.

The boundary layer scalings from the Boundary Layer Transition Tool documentation [12] are used to obtain a scaling for parameter (21): $M_{e}$ is not scaled with $R e, R e_{\theta}$ is scaled with $\sqrt{R e}$, and $\delta$ is scaled with $1 / \sqrt{R e}$. $\left(H_{e} / H_{w}\right)^{0.3}$ is shown in reference [12] to scale with $R e / 10$, and is neglectedmeaning it is unscaled in the present analysis - because the variation is an order of magnitude less than the variation of $R e_{\theta} / \delta$. Thus the parameter (21) is scaled with $R e$.

The linear scaling with Reynolds number is tested at Mach 19.4, by extrapolating the STS-107 parameter to the STS-115 conditions, a 16.7\% reduction in Reynolds number. Absolute values of the percentage differences between the computed and the extrapolated STS-115 parameter-(21) values that exceed $6.5 \%$ are shown as red in figure 4 . Approximately $95 \%$ of the windside surface has extrapolation errors less than $6.5 \%$.

The Reynolds number criterion for the allowable trajectory variation of the protuberance boundary layer trip parameter is

$$
\left(\frac{10}{6.5}\right) \times 16.7 \%=25.7 \% \simeq \pm 25 \%
$$

\section{6 $R e_{k}$ boundary layer transition parameter}

As part of the secondary correlation for predicting boundary layer transition induced by a protuberance, the Entry Aeroheating Subsystem team uses the parameter

$$
R e_{k}^{0.6}\left(R e_{\theta} \cdot \frac{\mu_{e}}{\mu_{k}}\right)^{0.4}
$$

All of the variables in parameter (23) are obtained from CFD solutions of the smooth-OML Orbiter.

As part of the Boundary Layer Transition Tool (version 2) documentation, a requirement of $11 \%$ accuracy to one standard deviation odds was placed on the CFD data [15]. Extrapolating this accuracy requirement to two-sigma, 


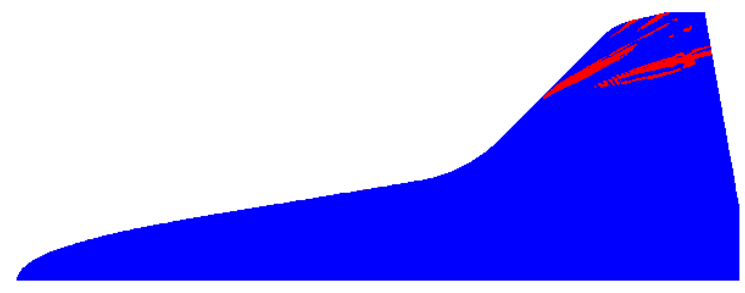

Figure 5. Absolute values of percentage extrapolation errors for $R e_{k}$ boundary layer transition parameter (23). Red is more than $4.5 \%$. Blue is less than $4.5 \%$.

or approximately 95\%, odds yields an accuracy requirement of approximately $20 \%$. The allowable error contribution due to trajectory variations is then set at $10 \%$.

Assuming $k \simeq \delta$, then both $R e_{k}$ and $R e_{\theta}$ can be scaled with $\sqrt{R e}$. Viscosity scales approximately with the square root of temperature, and so $\left(\mu_{e} / \mu_{k}\right)^{0.4}$ scales like $\left(T_{e} / T_{k}\right)^{0.2}$, and is neglected. Substitution into the expression (23) leads to the entire parameter being scaled with $\sqrt{R e}$.

The $\sqrt{R e}$ scaling is tested at Mach 19.4, by extrapolating the STS-107 parameter (23) to the STS-115 conditions, a $16.7 \%$ reduction in Reynolds number. Absolute values of the percentage differences between the computed and extrapolated STS-115 parameter- $(23)$ values that exceed $4.5 \%$ are shown as red in figure 5. Approximately $95 \%$ of the windside surface has extrapolation errors less than $4.5 \%$.

The Reynolds number criterion for the allowable trajectory variation of the $R e_{k}$ boundary layer trip parameter is

$$
\left(\frac{10}{4.5}\right) \times 16.7 \%=37.1 \% \simeq \pm 37 \%
$$

\subsection{Cavity-volume boundary layer transition parameter}

As part of the volume-based correlation for predicting boundary layer transition induced by a cavity, the Entry Aeroheating Subsystem team uses the parameter

$$
\frac{R e_{\theta}}{M_{e}} \cdot \frac{1}{\delta} \cdot\left(\frac{T_{e}}{T_{w}}\right)^{0.51}
$$

All of the variables in parameter (25) are obtained from CFD solutions of the smooth-OML Orbiter. 


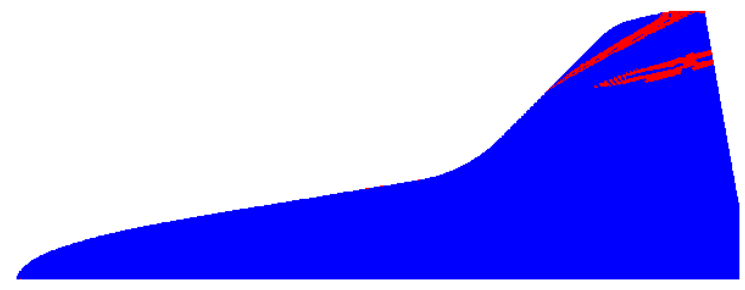

Figure 6. Absolute values of percentage extrapolation errors for cavity-volume boundary layer transition parameter (25). Red is more than $4 \%$. Blue is less than $4 \%$.

As part of the Boundary Layer Transition Tool (version 2) documentation, a requirement of $11 \%$ accuracy to one standard deviation odds was placed on the CFD data [15]. Extrapolating this accuracy requirement to two-sigma, or approximately 95\%, odds yields an accuracy requirement of approximately $20 \%$. The allowable error contribution due to trajectory variations is then set at $10 \%$.

$R e_{\theta}$ is scaled with $\sqrt{R e}, \delta$ is scaled inversely with $\sqrt{R e}$, and $M_{e}$ is unscaled. The temperature ratio scaling is neglected, because $T_{e} / T_{w}$ is shown to scale with $R e^{0.18}$ in reference [12], and when $T_{e} / T_{w}$ is raised to the 0.51 power the scaling becomes less than $R e^{0.1}$. The entire parameter (25) is thus scaled linearly with Reynolds number.

The linear scaling with Reynolds number is tested at Mach 19.4, by extrapolating the STS-107 parameter to the STS-115 conditions, a 16.7\% reduction in Reynolds number. Absolute values of the percentage differences between the computed and the extrapolated STS-115 parameter-(25) values that exceed $4 \%$ are shown as red in figure 6. Approximately $95 \%$ of the windside surface has extrapolation errors less than $4 \%$.

The Reynolds number criterion for the allowable trajectory variation of the cavity-volume boundary layer trip parameter is

$$
\left(\frac{10}{4}\right) \times 16.7 \%=41.8 \% \simeq \pm 40 \%
$$

\subsection{Cavity-area boundary layer transition parameter}

As part of the area-based correlation for predicting boundary layer transition induced by a cavity, the Entry Aeroheating Subsystem team uses the parameter

$$
\frac{R e_{\theta}}{M_{e}} \cdot \frac{1}{\delta} \cdot\left(\frac{T_{e}}{T_{w}}\right)^{0.67}
$$




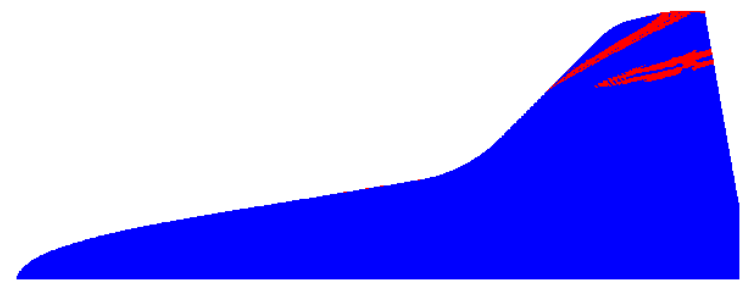

Figure 7. Absolute values of percentage extrapolation errors for cavity-area boundary layer transition parameter (27). Red is more than $4 \%$. Blue is less than $4 \%$.

All of the variables in parameter (27) are obtained from CFD solutions of the smooth-OML Orbiter.

As part of the Boundary Layer Transition Tool (version 2) documentation, a requirement of $11 \%$ accuracy to one standard deviation odds was placed on the CFD data [15]. Extrapolating this accuracy requirement to two-sigma, or approximately 95\%, odds yields an accuracy requirement of approximately $20 \%$. The allowable error contribution due to trajectory variations is then set at $10 \%$.

$R e_{\theta}$ is scaled with $\sqrt{R e}, \delta$ is scaled inversely with $\sqrt{R e}$, and $M_{e}$ is unscaled. The temperature ratio scaling is neglected because $T_{e} / T_{w}$ is shown to scale with $R e^{0.18}$ in reference [12], and when $T_{e} / T_{w}$ is raised to the 0.67 power the scaling becomes approximately $R e^{0.1}$. The entire parameter $(27)$ is thus scaled linearly with Reynolds number.

The linear scaling with Reynolds number is tested at Mach 19.4, by extrapolating the STS-107 parameter to the STS-115 conditions, a 16.7\% reduction in Reynolds number. Absolute values of the percentage differences between the computed and the extrapolated STS-115 parameter-(27) values that exceed $4 \%$ are shown as red in figure 7 . Approximately $95 \%$ of the windside surface has extrapolation errors less than $4 \%$.

The Reynolds number criterion for the allowable trajectory variation of the cavity-area boundary layer trip parameter is

$$
\left(\frac{10}{4}\right) \times 16.7 \%=41.8 \% \simeq \pm 40 \%
$$

\subsection{Boundary layer $C_{p}$, shock relations}

One of the techniques used by the Entry Aeroheating Subsystem team to estimate protuberance bending loads utilizes boundary-layer profile pressure coefficients that assume normal-shock stagnation pressures. These pressure 


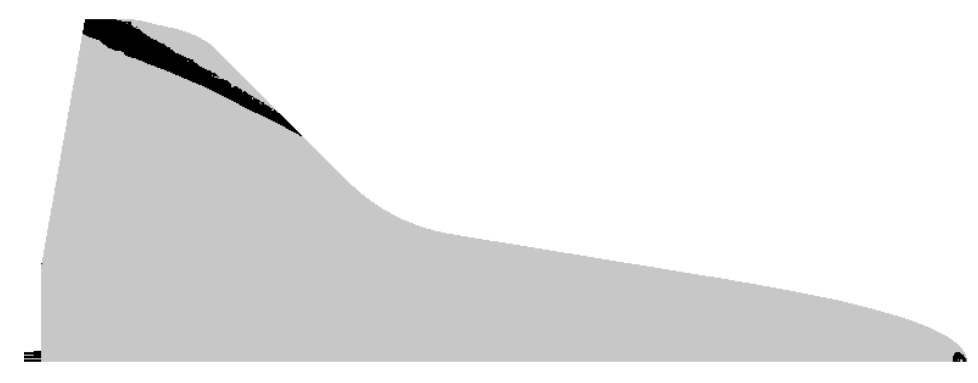

Figure 8. Absolute values of percentage errors for post normal-shock stagnation pressure coefficients. Black is more than $3 \%$.

coefficients are obtained from the smooth-OML CFD baseline solutions.

The bending loads process [16] assumes a $25 \%$ accuracy on the CFD pressures. The allowable error contribution due to trajectory variations is then set at $12 \%$.

To test the boundary layer pressure coefficient scaling, post normal-shock pressure coefficients are computed at the boundary layer edge for Mach-19.4 trajectory points on both the STS-107 and STS-115 trajectories. These two trajectory points differ by $16.7 \%$ in Reynolds number.

The absolute values of the percentage differences between the wind-side STS-115 and STS-107 pressure coefficients are shown in figure 8. Black regions have differences greater than $3 \%$. Approximately $95 \%$ of the wind-side surface has differences less than $3 \%$.

The Reynolds number criterion for the allowable trajectory variation of the normal-shock boundary layer pressure coefficients is

$$
\left(\frac{12}{3}\right) \times 16.7 \%=66.8 \% \simeq \pm 67 \%
$$

\subsection{Boundary layer $C_{p}$, stream-tube}

One of the techniques used by the Entry Aeroheating Subsystem team to estimate protuberance bending loads utilizes boundary-layer profile pressure coefficients that assume stream-tube theory total pressures, $P+\frac{1}{2} \rho V^{2}$. These pressure coefficients are obtained from the smooth-OML CFD baseline solutions.

The bending loads process [16] assumes a 25\% accuracy on the CFD pressures. The allowable error contribution due to trajectory variations is then set at $12 \%$.

To test the boundary layer pressure coefficient scaling, total-pressure coefficients are computed at the boundary layer edge for Mach-19.4 trajectory 


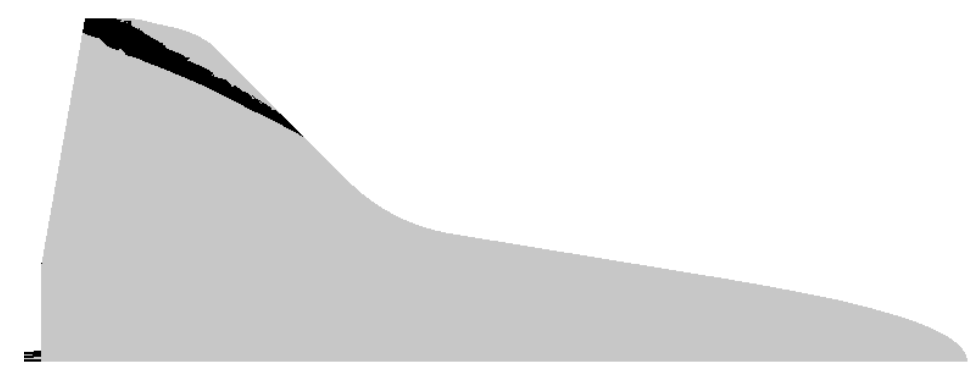

Figure 9. Absolute values of percentage errors for total-pressure coefficients. Black is more than $3 \%$.

points on both the STS-107 and STS-115 trajectories. These two trajectory points differ by $16.7 \%$ in Reynolds number.

The absolute values of the percentage differences between the wind-side STS-115 and STS-107 pressure coefficients are shown in figure 9. Black regions have differences greater than $3 \%$. Approximately $95 \%$ of the wind-side surface has differences less than $3 \%$.

The Reynolds number criterion for the allowable trajectory variation of the stream-tube boundary layer pressure coefficients is

$$
\left(\frac{12}{3}\right) \times 16.7 \%=66.8 \% \simeq \pm 67 \%
$$

\subsection{Displacement thickness}

The Entry Aeroheating Subsystem team has developed a tool, named Endigestion, for estimating breach flows. During operation, the Endigestion tool integrates mass flux through the boundary layer. The flow properties used by Endigestion are obtained from the smooth-OML CFD baseline solutions.

The estimate of the required CFD accuracy used during Endigestion development is $\pm 100 \%$. The allowable error contribution due to trajectory variations is then set at $50 \%$.

The displacement thickness is used to estimate the trajectory error scaling of the Endigestion mass flux. The displacement thickness scales with $1 / \sqrt{R e}$.

The STS- $107 \delta^{*}$ at $M_{\infty}=19.4$ are scaled to the STS- 115 trajectory, a $16.7 \%$ $R e$ difference. The absolute values of the percentage scaling errors are shown in figure 10, where the red indicates errors greater than $\pm 5 \%$. Approximately $95 \%$ of the wind-side surface has scaling errors less than $5 \%$.

The Reynolds number criterion for the allowable trajectory variation of the displacement thickness is

$$
\left(\frac{50}{5}\right) \times 16.7 \%=167 \% \simeq \pm 165 \%
$$




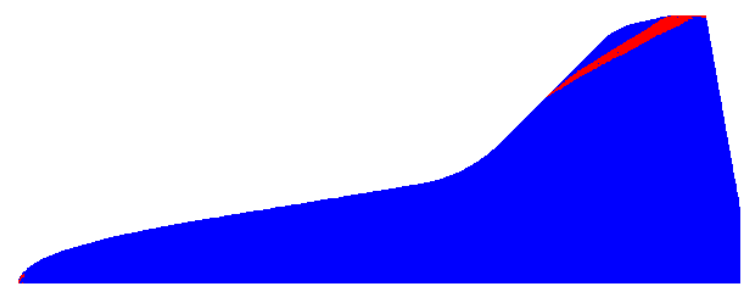

Figure 10. Absolute values of percentage errors for $\delta^{*}$ scaling. Red is more than $5 \%$. Blue is less than $5 \%$.

\subsection{Total enthalpy ratio}

The Entry Aeroheating Subsystem team has developed a tool, named Endigestion, for estimating breach flows. The Endigestion tool estimates the fraction of free stream total enthalpy that is ingested into the breach. The flow properties used by Endigestion are obtained from the smooth-OML CFD baseline solutions.

The estimate of the required CFD accuracy used during Endigestion development is $\pm 100 \%$. The allowable error contribution due to trajectory variations is then set at $50 \%$.

The scaling of the total enthalpy ratio $H_{w} / H_{e}$ is used here to estimate the trajectory scaling error for the Endigestion total enthalpy ratio. As part of the BLT (version 2) documentation [12], the percentage change in the ratio $H_{w} / H_{e}$ is shown to scale as -0.4 times the percentage change in Reynolds number for $M_{\infty} \geq 20$. In reference [12], this scaling has been normalized for a $10 \%$ change in Reynolds number. Figure 11 re-plots the scaled data from reference [12], this time showing a threshold of $\pm 3 \%$ error in the scaling, marked red in the figure. Over $95 \%$ of the wind side surface is seen to have a scaling error less than $3 \%$.

The Reynolds number criterion for the allowable trajectory variation of the total enthalpy ratio is

$$
\left(\frac{50}{3}\right) \times 10 \%=167 \% \simeq \pm 165 \%
$$

\subsection{Lower priority data types}

The Entry Aeroheating Subsystem team has determined allowable trajectory errors due to trajectory variations for five more types of CFD data. But, Reynolds number criteria were not developed for them. These data types have been considered by the Entry Aeroheating Subsystem team to be of lower priority than the twelve previously discussed types. 


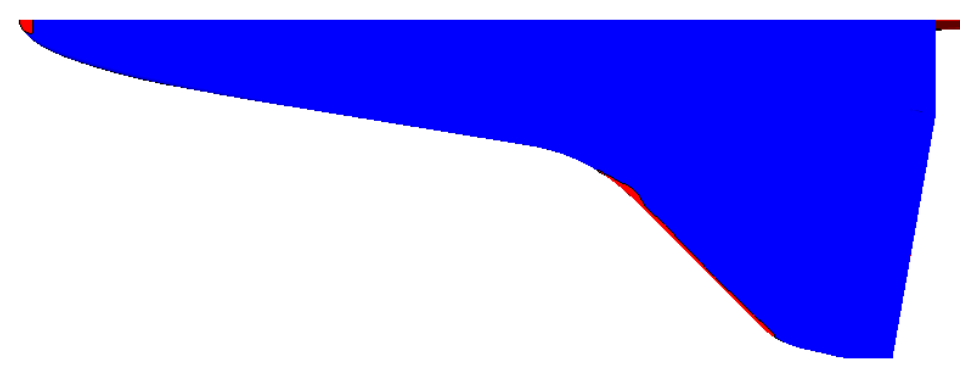

Figure 11. Absolute values of percentage errors from scaling the percentage changes in $H_{w} / H_{e}$ by -0.4 times the percentage change in Reynolds number. Red is more than $3 \%$. Blue is less than $3 \%$.

Pressure coefficients on protrusion surfaces obtained from CFD solutions on the actual protrusion geometry have the same allowable trajectory errors as developed in sections 4.9 and 4.10 . Those sections established a $25 \%$ allowable total CFD accuracy, and a $12 \%$ allowable error due to trajectory variation.

Wing leading-edge protrusion heating bump factors, such as RCC plug repairs or RCC crack repairs with the NOAX material, are assumed to have an uncertainty comparable to the cavity heating bump factors from section 4.1. The total CFD uncertainty is estimated at 50\%, and the allowable error due to trajectory variations is set at $25 \%$.

General wing leading-edge heating rates from CFD predictions are estimated to have a $40 \%$ accuracy, based upon undocumented comparisons made for the Orbiter body point 5505 to radiometer flight data. Those informal comparisons were made as part of the X-37 program. Hence, the allowable error due to trajectory variations is set at $20 \%$.

Limited lee-side heating rate comparisons between CFD and Orbiter flight data were made in reference [17]. The comparisons were with thermocouple readings from STS-2, STS-3, and STS-5, and with the Shuttle Infrared Leeside Temperature Sensing (SILTS) [18] flight experiment data. The CFD accuracy for lee-side heating rates was shown to be approximately $60 \%$. The allowable error due to trajectory variations is then set at $30 \%$.

A spot check of wind-side turbulent heating rates between CFD and STS-28 thermocouple measurements was made by the Entry Aeroheating Subsystem team during the STS-121 mission. That limited comparison showed a $40 \%$ accuracy for the CFD predictions. Hence, the allowable error due to trajectory variations is set at $20 \%$. 


\begin{tabular}{clccc} 
Priority & Parameter & $\begin{array}{c}\text { allowable } \\
\text { uncertainty } \\
\text { estimate, } \%\end{array}$ & $\begin{array}{c}\text { trajectory } \\
\text { error, } \%\end{array}$ & $\begin{array}{c}\text { trajectory } R e \\
\text { criterion, } \%\end{array}$ \\
\hline 1 & Cavity heating bump factors & 50 & 25 & 31 \\
2 & Smooth OML temperatures & 6 & 3 & 32 \\
3 & Smooth OML heating rates & 20 & 10 & 30 \\
4 & Boundary layer thickness & 22 & 11 & 40 \\
5 & $\frac{R e_{\theta}}{M_{e}} \cdot \frac{1}{\delta} \cdot\left(\frac{H_{e}}{H_{w}}\right)^{0.3}$ & 20 & 10 & 25 \\
6 & $R e_{k}^{0.6}\left(R e_{\theta} \cdot \frac{\mu_{e}}{\mu_{k}}\right)^{0.4}$ & 20 & 10 & 37 \\
7 & $\frac{R e_{\theta}}{M_{e}} \cdot \frac{1}{\delta} \cdot\left(\frac{T_{e}}{T_{w}}\right)^{0.51}$ & 20 & 10 & 40 \\
8 & $\frac{R e_{\theta}}{M_{e}} \cdot \frac{1}{\delta} \cdot\left(\frac{T_{e}}{T_{w}}\right)^{0.67}$ & 20 & 10 & 40 \\
9 & Boundary layer $C_{p},($ shock $)$ & 25 & 12 & 67 \\
10 & Boundary layer $C_{p},($ stream-tube) & 25 & 12 & 67 \\
11 & Displacement thickness & 100 & 50 & 165 \\
12 & $\frac{H_{w}}{H_{e}}$ & 100 & 50 & 165 \\
13 & Protruding gap filler $C_{p}$ & 25 & 12 & \\
14 & RCC plug heating bump factors & 50 & 25 & \\
15 & Wing leading-edge heating rates & 40 & 20 & \\
16 & Lee-side heating rates & 60 & 30 & \\
17 & Turbulent heating rates & 40 & 20 &
\end{tabular}

Table 3. Summary of estimated uncertainty for CFD products, the allowable error contributions due to trajectory variations as set within the present report, and the resulting trajectory variation limits expressed as Reynolds number thresholds.

\subsection{Tabulation}

Table 3 lists the estimated CFD uncertainty and/or accuracy for each of the 17 CFD products discussed in the present report, along with the allowable error contribution due to trajectory variations. For the 12 highest-priority products, the last column of table 3 lists the resulting allowable trajectory variation, expressed in terms of trajectory Reynolds number. The most restrictive criterion, for the primary protuberance-induced boundary layer transition parameter, allows a $\pm 25 \%$ variation in trajectory Reynolds number.

\section{Summary of results}

Criteria were established for reusing the computational fluid dynamics (CFD) solutions of the Space Shuttle Orbiter at specific entry trajectory points for new entry trajectories. These criteria were developed for 12 particular types of aeroheating data that the Entry Aeroheating Subsystem team might need 
during a Space Shuttle mission to assess thermal protection damage to the Orbiter. The criteria are expressed in terms of Reynolds number variations for fixed Mach numbers.

The development of the trajectory variation criteria began by defining either an estimated uncertainty or an accuracy requirement for each of the 17 CFD aeroheating datum types, assuming no trajectory errors. Then the allowable error contribution due to trajectory variations was set to be half of the previously established CFD uncertainty value. For the 12 highest priority of the 17 datum types, scaling relations were applied to cast the datum uncertainty in terms of the trajectory Reynolds number variations. Spot checks of the scalings were performed against the CFD solutions for different trajectories, and the allowable trajectory variation criteria were obtained to approximately 95\%-confidence levels.

The criteria were developed under several assumptions: a spot-check at one Mach number applied for all Mach numbers (the Mach numbers of most interest were greater than 15); uncertainties were the same for the positive and negative sides; uncertainty trends extrapolated linearly in Reynolds number; and, angle of attack variations were negligible (the angles of attack were within $40 \pm 2^{\circ}$ ).

The most restrictive trajectory variation criteria, for a protuberance-induced boundary layer transition correlation parameter, allows for a $25 \%$ variation in Reynolds number. The criteria for all the eight highest-priority datum types allow $40 \%$ or less variations in Reynolds number. In light of the noted development assumptions, for future Space Shuttle missions the Entry Aeroheating Subsystem team has decided to use a criteria of 15-20\% variation in Reynolds number, when assessing the need for trajectory-specific CFD solutions.

\section{References}

1. External Aerothermal Analysis Team: Smooth Outer Mold Line Aerothermal Solution Database for Orbiter Windside Acreage Environments During Nominal Entry Conditions. Engineering Note EG-SS-06-1, NASA Johnson Space Center, Houston, Texas, Apr. 2005. Presented to Orbiter Configuration Control Board.

2. Campbell, C. H.; Anderson, B.; Bourland, G.; Bouslog, S.; Cassady, A.; Horvath, T. J.; Berry, S. A.; Gnoffo, P. A.; Wood, W. A.; Reuther, J. J.; Driver, D. M.; Chao, D. C.; Hyatt, J.; and Picetti, D.: Orbiter Return to Flight Entry Aeroheating. AIAA Paper 2006-2917, June 2006.

3. Reuther, J.; Thompson, R.; Pulsonetti, M.; and Campbell, C.: Computational Aerothermodynamic Analysis for the STS-107 Accident Investigation. AIAA Paper 2004-1384, Jan. 2004. 
4. Wright, M. J.; Candler, G. V.; and Bose, D.: Data-Parallel Line Relaxation Method for the Navier-Stokes Equations. AIAA Journal, vol. 36, no. 9, Sept. 1998, pp. 1603-1609.

5. Gnoffo, P. A.; Gupta, R. N.; and Shinn, J. L.: Conservation Equations and Physical Models for Hypersonic Air Flows in Thermal and Chemical Nonequilibrium. NASA TP 2867, Feb. 1989.

6. Gnoffo, P. A.: An Upwind-Biased, Point-Implicit Relaxation Algorithm for Viscous, Compressible Perfect-Gas Flows. NASA TP 2953, Feb. 1990.

7. Navier, M.: Mémoire sur les lois du Mouvement des Fluides. Mémoire de l'Académie des Sciences, vol. 6, 1827, p. 389.

8. Stokes, G. G.: On the Theories of the Internal Friction of Fluids in Motion. Trans. Cambridge Philosophical Society, vol. 8, 1849, pp. 227-319.

9. Pulsonetti, M. V.; and Wood, W. A.: Computational Aerothermodynamic Assessment of Space Shuttle Orbiter Tile Damage - Open Cavities. AIAA Paper 2005-4679, June 2005.

10. Hyatt, J.; Wang, K. C.; Everhart, J.; Greene, F.; Merski, R.; Wood, B.; Berger, K.; Anderson, B.; and Cassady, A.: The Cavity Heating Tool. Technical Memorandum ATA-AH-TM-2006-035, The Boeing Company, Houston, Texas, Mar. 2006.

11. Palmer, G.; Alter, S.; Everhart, J.; Wood, W.; Driver, D.; J.Brown; and Prabhu, R.: CFD Validation for Long and Short Cavity Flow Simulations. AIAA Paper 2007-4254, June 2007.

12. Wood, W. A.; Alter, S. J.; Driver, D. M.; Meyer, B.; Greene, F. A.; Saunders, D. A.; Prabhu, D. K.; Mazaheri, A. R.; Prabhu, R. K.; and Calloway, R.: Viscous-CFD Space Shuttle Orbiter Boundary Layer Data and Probe Software. Engineering Note EG-SS-07-10, NASA Johnson Space Center, Houston, Texas, Mar. 2007.

13. Saunders, D.; and Prabhu, D.: Curvature-Based Boundary Layer Edge Detection. In preparation, Eloret Corporation, 2008.

14. Schlichting, H.: Boundary-Layer Theory. McGraw-Hill Book Company, New York, seventh ed., 1979.

15. Wood, W. A.; Prabhu, R. K.; Calloway, R.; and Mazaheri, A. R.: Requirement Verification for Viscous-CFD Space Shuttle Orbiter Boundary Layer Data and Probe Software. Engineering Note EG-SS-07-11, NASA Johnson Space Center, Houston, Texas, Mar. 2007. 
16. Campbell, C. H.; Driver, D. M.; Alter, S. J.; Fasanella, E. L.; Wood, W. A.; and Stone, J. S.: Orbiter Gap Filler Bending Model for Re-entry. AIAA Paper 2007-413, Jan. 2007.

17. Weilmuenster, K. J.; and Gnoffo, P. A.: Solution Strategies and Heat Transfer Calculations for Three-Dimensional Configurations at Hypersonic Speeds. AIAA Paper 92-2921, July 1992.

18. Throckmorton, D. A.; Zoby, E. V.; and Kantsios, A. G.: The Shuttle Infrared Leeside Temperature Sensing (SILTS) Experiment. AIAA Paper 1985-328, Jan. 1985. 


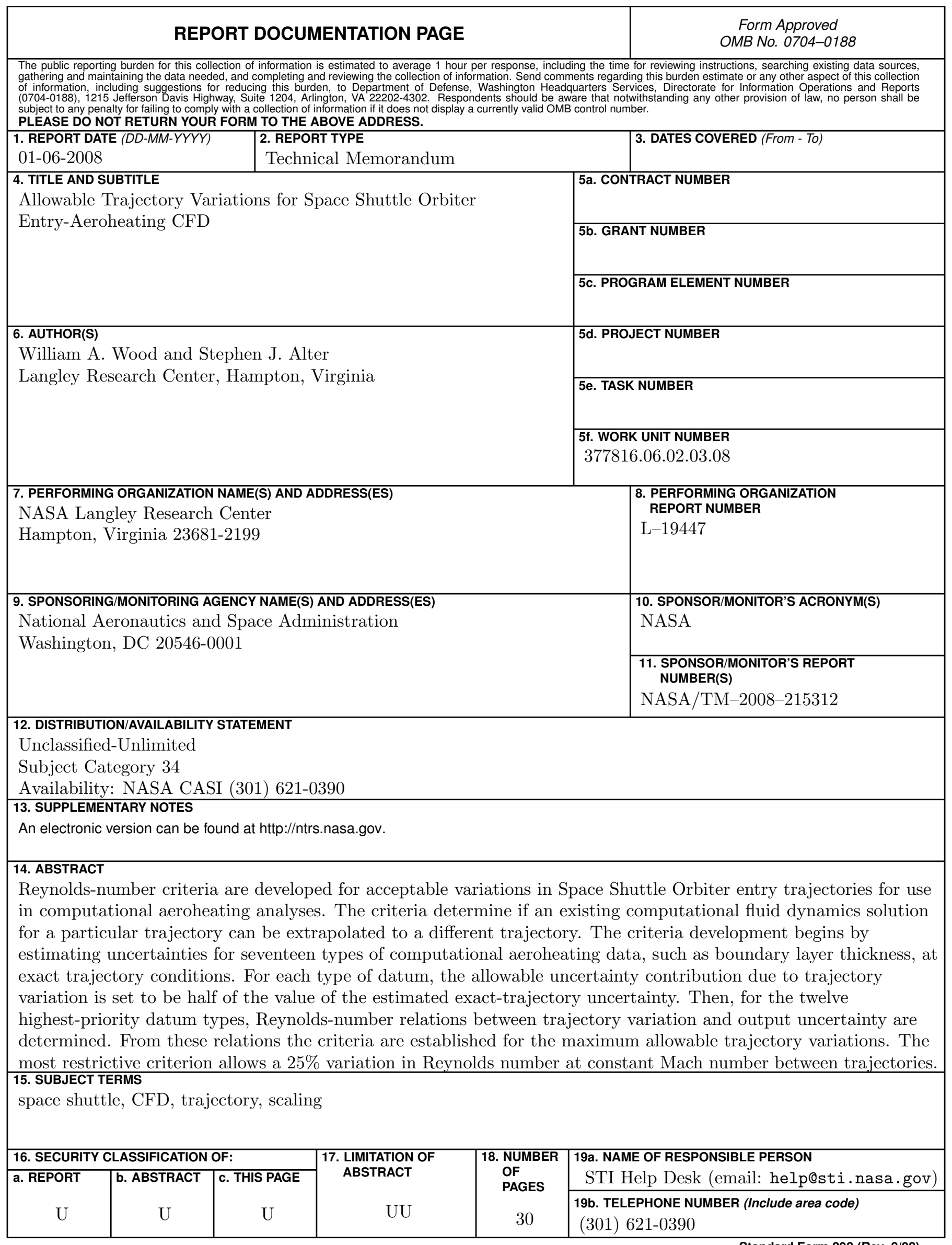



